\title{
A randomised study of comfort during bronchoscopy comparing conscious sedation and anaesthetist-controlled general anaesthesia, including the utility of bispectral index monitoring
}

\author{
Thomas R. Skinner (1) 1 , Joseph Churton ${ }^{1}$, Timothy P. Edwards ${ }^{1}$, \\ Farzad Bashirzadeh ${ }^{1}$, Christopher Zappala ${ }^{1}$, Justin T. Hundloe ${ }^{1}$, Hau Tan $^{2}$, \\ Andrew J. Pattison ${ }^{1}$, Maryann Todman ${ }^{1}$, Gunter F. Hartel $\mathbb{1}^{3}$ and \\ David I. Fielding (1) ${ }^{1}$
}

Affiliations: 'Dept of Thoracic Medicine, Royal Brisbane and Women's Hospital, Herston, QLD, Australia. ${ }^{2}$ Dept of Anaesthetic Services, Royal Brisbane and Women's Hospital, Herston, QLD, Australia. ${ }^{3}$ Statistics Unit, QIMR Berghofer Medical Research Institute, Herston, QLD, Australia.

Correspondence: Thomas R. Skinner, Thoracic Medicine, Royal Brisbane and Women's Hospital, Butterfield St, Herston, Brisbane, QLD, 4029, Australia. E-mail: Thomas.skinnerahealth.qld.gov.au

\section{ABSTRACT}

Background: The difference in patient comfort with conscious sedation versus general anaesthesia for bronchoscopy has not been adequately assessed in a randomised trial. This study aimed to assess if patient comfort during bronchoscopy with conscious sedation is noninferior to general anaesthesia.

Methods: 96 subjects were randomised to receive conscious sedation or general anaesthesia for bronchoscopy. The primary outcome was subject comfort. Secondary outcomes included willingness to undergo a repeat procedure if necessary and level of sedation assessed clinically and by bispectral index (BIS) monitoring.

Results: There was no significant difference between subject comfort scores (difference $-0.01,95 \%$ CI $-0.63-0.61$ on a 10 -point scale; $\mathrm{p}=0.97)$ or willingness to undergo a repeat procedure $(97.7 \%$ versus $91.8 \%$, 95\% CI $-4.8-15.5 \%$; $\mathrm{p}=0.37$ ). Deeper levels of sedation in the general anaesthesia cohort was confirmed with both clinical and BIS monitoring. There was no significant difference in diagnostic accuracy (conscious sedation 93.9\%, 95\% CI 80.4-98.3\% versus general anaesthesia 86.5\%, 95\% CI 72.0-94.1\%; $\mathrm{p}=0.43$ ). There were more complications $(29.6 \%$, 95\% CI $18.2-44.2 \%$ versus $6.1 \%$, 95\% CI $2.1-16.5 \%$; $\mathrm{p}<0.01)$ in the general anaesthesia group. There was no relationship between high BIS scores and subject discomfort. BIS levels $<40$ during a procedure were associated with increased complications.

Conclusion: Conscious sedation is not inferior to general anaesthesia in providing patient comfort during bronchoscopy, despite lighter sedation, and is associated with fewer complications and comparable diagnostic accuracy. BIS monitoring may have a role in preventing complications associated with deeper sedation.

\section{@ERSpublications}

Conscious sedation was noninferior to anaesthetist-controlled general anaesthesia for patient comfort during bronchoscopy in this randomised trial. Deeper sedation with general anaesthesia did not improve comfort and had increased complications. https://bit.ly/3sCDfEy

Cite this article as: Skinner TR, Churton J, Edwards TP, et al. A randomised study of comfort during bronchoscopy comparing conscious sedation and anaesthetist-controlled general anaesthesia, including the utility of bispectral index monitoring. ERJ Open Res 2021; 7: 00895-2020 [https:// doi.org/10.1183/23120541.00895-2020].

This article has supplementary material available from openres.ersjournals.com

This study is registered at www.anzctr.org.au with identifier number ACTRN12621000358842. Individual deidentified patient data will be available upon reasonable request with the corresponding author or through the Dryad digital repository (https://doi.org/10.5061/dryad.j3tx95xd6). This will be immediately following publication.

Received: 1 Dec 2020 | Accepted: 22 Feb 2021

Copyright $\odot$ The authors 2021. This version is distributed under the terms of the Creative Commons Attribution NonCommercial Licence 4.0. For commercial reproduction rights and permissions contact permissions@ersnet.org 


\section{Introduction}

Ideal sedation for bronchoscopy should allow optimal patient comfort and not compromise diagnostic accuracy or increase the risk of complications [1-8]. Conscious sedation offers flexibility by being able to be administered by a physician, compared with deep sedation and general anaesthesia, which require an anaesthetist [9]. This may reduce costs. The 2013 British Thoracic Society guidelines for diagnostic bronchoscopy suggest the desired depth of sedation is usually conscious sedation [7]. However, in current practice the sedation strategy employed for bronchoscopy varies widely based on local practices and policy [10-13]. Recent Australian data highlight this variability, with anaesthetists present for $38 \%$ of procedures in the public setting and $81 \%$ of those performed privately, as well as significantly differing anaesthesia strategies [13].

Clinicians' understanding of patient comfort is crucial to appropriate care of patients. Prior studies have not assessed comfort as a primary outcome in a randomised prospective study of bronchoscopy, rather only reporting comfort as a secondary outcome or using a limited binary (distress versus no distress) assessment [14-16]. In addition, the impressions of patient comfort of other procedural staff, including bronchoscopy nurses, is important.

Recent papers have shown the diagnostic accuracy of flexible bronchoscopy and endobronchial ultrasound (EBUS) transbronchial needle aspiration (TBNA) with conscious sedation is noninferior to deep sedation or general anaesthesia [15-23]. Trials assessing complications with conscious sedation versus general anaesthesia have conflicting results, and this appears to be due to differing definitions of complications [14-16].

Bispectral index (BIS) monitoring can be used to objectively assess sedation levels during procedures [24]. This involves the conversion of a processed electroencephalogram into a numbered index ranging from 0 to 100 , with 100 being fully awake and 40-60 considered appropriate for general anaesthesia in bronchoscopy [25]. Studies have demonstrated correlation between BIS and the modified Observer's Assessment of Alertness and Sedation (mOAA/S) scale, including in endoscopy [26-28]. However, this relationship has not been consistent, meaning its role is predominantly supplementary at this time [29-31]. No studies have analysed BIS with differing sedation types at bronchoscopy [25, 32, 33]. Therefore, BIS may have a role in monitoring patient comfort given that amnestic effects of sedation mean patients may not recall discomfort afterwards.

We conducted a randomised clinical trial aiming to assess for noninferiority in subject comfort during bronchoscopy with conscious sedation versus general anaesthesia. Secondary end-points were observer-perceived comfort and sedation scores (by both doctors and nurses), depth of sedation as recorded by BIS, personnel requirement, complication rates and diagnostic accuracy.

\section{Methods}

\section{Subjects and procedure}

This randomised prospective trial was conducted within a tertiary hospital from October to December 2013 and from July to December 2014. These dates were used to allow key study personnel to be present. The study cohort consisted of all patients scheduled for either standard bronchoscopy (with or without endobronchial or transbronchial sampling), EBUS TBNA or radial-probe EBUS biopsy. All subjects gave written informed consent prior to participating in the study. Exclusion criteria were those aged $<18$ years, pregnant women, those with an artificial airway such as tracheostomy, allergy requiring specific anaesthesia and those unable to give accurate information regarding their experience (i.e. those lacking mental capacity). In addition, we excluded those specifically requesting general anaesthesia, those likely to experience severe cough or where the expected procedure time fell outside the scope of conscious sedation. Subjects were excluded if they were not available to undergo the procedure on alternative days, i.e. with or without anaesthetic support. This was the case for subjects who were acutely unwell or were required to travel significant distances.

All procedures were performed within a standalone bronchoscopy suite with full anaesthetic monitoring equipment. BIS monitoring bands were applied according to manufacturer's specification prior to the procedure for all subjects.

Conscious sedation was performed by two proceduralists with oral bronchoscope insertion via a bite block. No anaesthetist was present. One of the two bronchoscopy proceduralists was responsible for delivery of sedation and monitoring cardiorespiratory parameters. Both fentanyl and midazolam were used, titrated by the proceduralist to effect, up to a maximum of $0.1 \mathrm{mg}$ and $5 \mathrm{mg}$, respectively. Deep sedation and general anaesthesia were performed by an anaesthetist using a laryngeal mask airway; choice of anaesthetic agents was at the discretion of the anaesthetist. While recognising the clear differences between deep sedation and general anaesthesia, we refer to all anaesthetist-controlled procedures as general anaesthesia. 
Randomisation and blinding

Subjects were randomised prior to any anaesthetic review to either conscious sedation or general anaesthesia. Allocation was by using sequentially numbered opaque envelopes stratified for the subjects' comorbidities using the Charlson score. The procedure type was not blinded; however, both procedural and anaesthetic staff were blinded to the BIS monitoring data during the procedure.

\section{Subject and proceduralist survey}

Post-procedural surveys were answered during a phone consultation $24-48 \mathrm{~h}$ post-procedure. The survey assessed tolerability of the procedure on a Likert scale of 1 (tolerable) to 10 (intolerable), as well as subject willingness to undergo a repeat procedure if necessary, i.e. yes or no (appendix B). In addition, pre(collected on the day of procedure, face-to-face) and post-procedure emotional state was assessed in surveys, to assess for any differences (appendices A and B). The emotional state score was calculated by subtracting the negative emotion scores (tense, upset and worried) from the positive emotion scores (calm, relaxed and content). Additionally, both the primary proceduralist and the nurse assistant were asked to rate their perception of the subject's comfort on the same Likert and willingness scales, as well as the level of sedation according to the mOAA/S scale (appendix C). Results of the bronchoscopy were not known to the subject at the time of follow-up phone call.

\section{Study end-points and statistical analysis}

The primary end-point was subject comfort assessed by a 10-point Likert scale. A sample size estimate of 44 was calculated with a 1.5-points noninferiority limit (for a 10-point scale) between sedation methods for the primary end-point of comfort, assuming an $\alpha$-level of 0.05 and a $5 \%$ dropout rate based on data from Clark et al. [34]. Since the post-procedure questionnaire was reported by subjects by phone, it wasn't possible to use a visual analogue scale (VAS); therefore, the 10-point Likert was chosen to approximate the 1-100-mm VAS.

The secondary end-points included willingness to undergo a repeat procedure, pre- and post-procedure subject emotional state, proceduralist and nurse assistant assessment of comfort and sedation, depth of anaesthesia as measured by BIS, procedure duration, staff number, complications, diagnostic yield and diagnostic accuracy.

Blinding meant that BIS was not actively monitored to adjust sedation in the procedures. To be included in the assessment, BIS data for $>60 \%$ of the procedure was required. Time and percentage of total time with a BIS level $<40,40-60,60-80$ and $>80$ were assessed.

Complications recorded were defined in appendix D. Diagnostic yield was assessed for all diagnostic procedures including diagnostic accuracy for malignancy (defined in appendix D). Subjects were followed for $\geqslant 1$ year to determine final diagnosis. When the bronchoscopy result was not positive for malignancy and 1 year of follow-up data was unavailable, subjects were excluded from this portion of the analysis.

Quantitative variables are summarised as mean \pm SD and differences between the treatment groups with $95 \%$ confidence intervals. Comparisons were performed with the t-test, Chi-squared or Fisher's exact test, as appropriate, for comparisons of qualitative variables. All tests were two-sided, and a p-value $<0.05$ was considered to be statistically significant. Assessments of subject comfort were compared using matched-pairs analyses and Pearson's correlation. Comparison of perceived willingness to repeat the procedure was performed using Bowker's test of symmetry. Time spent at different BIS levels was analysed using repeated-measures random-effects models. Percentage of time with BIS $>80$ and proceduralist and nurse assessments of sedation were evaluated by regression analysis.

\section{Statement of ethics}

The research was conducted ethically in accordance with the World Medical Association Declaration of Helsinki. All subjects gave written informed consent, and the study was approved by the Royal Brisbane and Women's Hospital human research ethics committee (reference number: HREC/13/QRBW/145).

\section{Results}

During the study period, 260 patients were considered for enrolment, and 96 were available for randomisation (figure 1). The commonest reason for exclusion was nonavailability of the patients on the 2 days of the week when anaesthetists were present if necessary after randomisation. 50 subjects were allocated to the conscious sedation arm, while 46 were allocated to the general anaesthesia arm. One and two subjects were lost to follow-up in the conscious sedation arm and the general anaesthesia arm, respectively. 


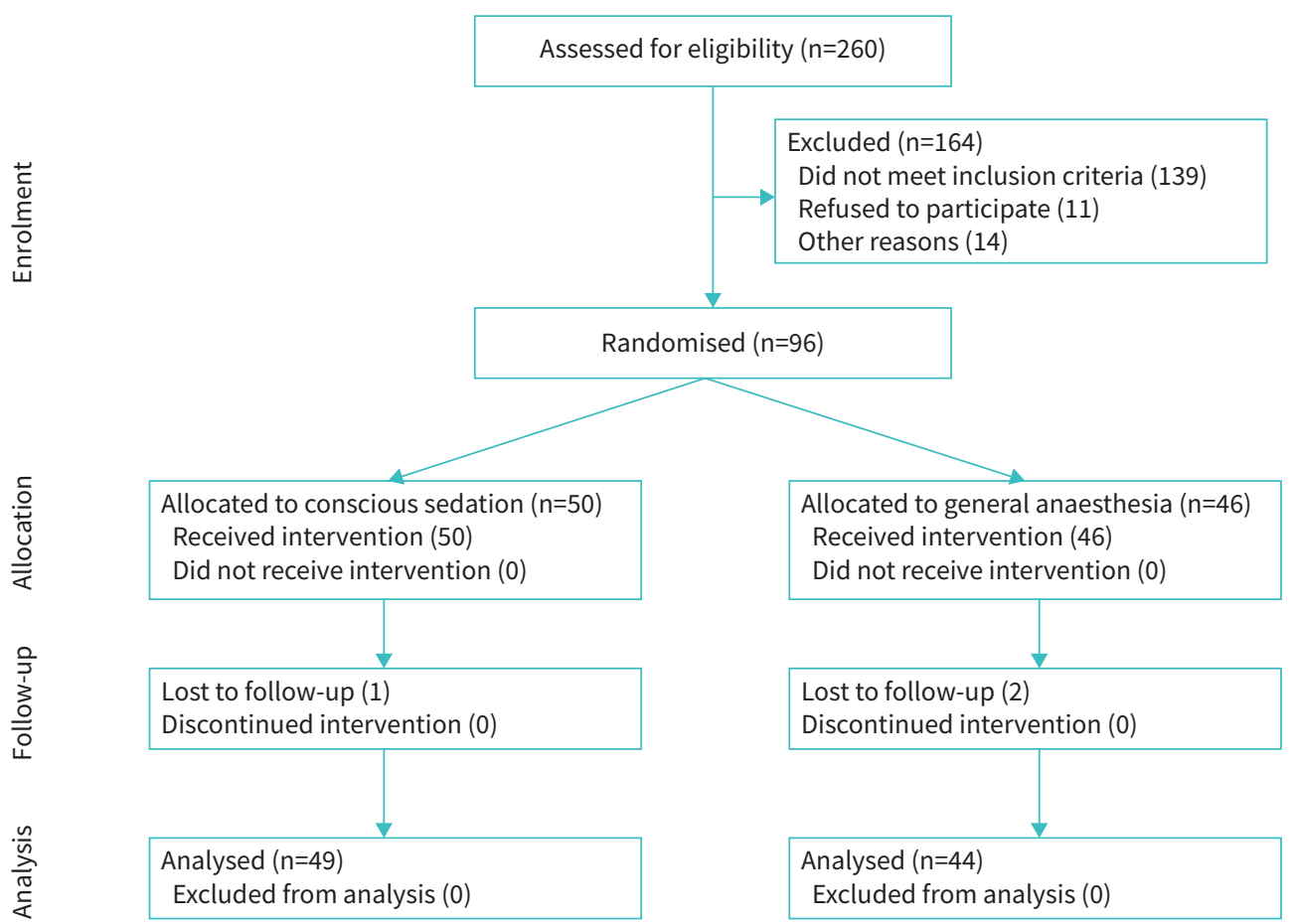

FIGURE 1 Consolidated Standards of Reporting Trials diagram of participant enrolment, allocation, follow-up and analysis.

Characteristics of the treatment groups are presented in table 1. The were no significant differences in age, sex, Charlson comorbidity index, pre- and post-procedure emotional state score (suggesting comparable levels of peri-procedural anxiety) or final diagnoses between the groups. Both groups were comparable in terms of procedure type and comorbidities.

All conscious sedation procedures received fentanyl and midazolam at mean \pm SD of $90.6 \pm 16.0 \mu \mathrm{g}$ and $4.4 \pm 1.0 \mathrm{mg}$, respectively. General anaesthesia strategies most commonly involved opioids (81.0\%), propofol (69.0\%) and midazolam (66.7\%).

\section{Subject comfort}

Table 2 summarises the primary outcome of comfort scores as reported by the subjects, as well as how proceduralists and nursing staff perceived the subject's experience. The mean comfort score as reported by subjects was $2.2 \pm 1.57$ and $2.2 \pm 1.38$ (difference $-0.01,95 \% \mathrm{CI}-0.63--0.61$ ) on a 10 -point scale, satisfying the \pm 1.5 noninferiority criterion; $p=0.97)$ in the conscious sedation and general anaesthesia groups, respectively. Willingness to undergo a repeat procedure was reported by all but five subjects; $97.7 \%$ of conscious sedation subjects and $91.8 \%$ of general anaesthesia subjects (difference $5.8 \%, 95 \%$ CI $-4.8-15.5 \%$; $=0.37$ ).

\section{Perceptions of subject comfort}

Subject comfort, as perceived by proceduralists, was not significantly different between the conscious sedation and general anaesthesia groups $(\mathrm{p}=0.16)$. Proceduralists' assessment of subject comfort was similar to subject's self-assessment (matched-pairs analysis, mean difference $0.10,95 \% \mathrm{CI}-0.36-0.56$; $\mathrm{p}=0.67$ ) (figure 2a). However, proceduralist perception was not strongly correlated with that of the subjects' self-rating (Pearson correlation $\mathrm{r}=0.18$, 95\% CI $-0.028-0.37 ; \mathrm{p}=0.09$ ) (figure 2a). Subject comfort as perceived by nurses was worse with conscious sedation $(p=0.05)$. Furthermore, nurses tended to overestimate subjects' discomfort rating relative to the subjects' self-ratings (matched-pairs analysis, mean difference $0.98,95 \%$ CI $0.41-1.54 ; \mathrm{p}<0.01$ ) (figure $2 \mathrm{~b}$ ). However, the nurses' perception correlated with the subjects' self-rating more closely (Pearson correlation $\mathrm{r}=0.34,95 \%$ CI $0.15-0.51 ; \mathrm{p}<0.01$ ). Despite this, nurse and proceduralist ratings were most highly correlated with each other (Pearson correlation $\mathrm{r}=0.70$, 95\% CI 0.58-0.79; $\mathrm{p}<0.01)$. 


\begin{tabular}{|c|c|c|c|}
\hline & $\begin{array}{l}\text { Conscious } \\
\text { sedation }\end{array}$ & $\begin{array}{c}\text { General } \\
\text { anaesthesia }\end{array}$ & p-value \\
\hline All procedures & 49 & 44 & \\
\hline Age years & $58.2 \pm 13.7$ & $61.3 \pm 14.0$ & 0.28 \\
\hline Female & 19 (38.8) & 12 (27.3) & 0.34 \\
\hline Charlson comorbidity index & & & 0.86 \\
\hline $0-1$ (low) & $23(46.9)$ & $19(43.2)$ & \\
\hline 2-3 (moderate) & $11(22.4)$ & $11(25.0)$ & \\
\hline$\geqslant 4$ (high) & $12(24.5)$ & $14(31.8)$ & \\
\hline \multicolumn{4}{|l|}{ Emotional state score } \\
\hline Pre-procedure & $4.6 \pm 3.4$ & $4.7 \pm 3.9$ & 0.91 \\
\hline Post-procedure & $2.4 \pm 1.5$ & $2.5 \pm 1.6$ & 0.79 \\
\hline Procedure & & & 0.23 \\
\hline $\begin{array}{l}\text { Standard bronchoscopy (not for investigation of } \\
\text { malignancy) }\end{array}$ & 6 & 2 & \\
\hline $\begin{array}{l}\text { Standard bronchoscopy (for investigation of } \\
\text { malignancy) }\end{array}$ & 8 & 4 & \\
\hline TBLB & 6 & 4 & \\
\hline Convex EBUS TBNA & 12 & 13 & \\
\hline Radial EBUS guide sheath biopsy & 13 & 15 & \\
\hline Combined: TBLB/EBUS TBNA & 1 & 1 & \\
\hline Combined: EBUS TBNA/EBUS guide sheath & 1 & & \\
\hline Therapeutic bronchoscopy & 2 & 5 & \\
\hline All diagnostic procedures & 47 & 39 & \\
\hline Final diagnosis: malignant & $13(27.7)$ & $18(46.2)$ & 0.11 \\
\hline Nonsmall cell lung cancer & 13 & 13 & \\
\hline Small cell lung cancer & 0 & 0 & \\
\hline Lymphoma & 0 & 2 & \\
\hline Metastatic malignancy & 0 & 3 & \\
\hline Final diagnosis: nonmalignant & 34 (72.3) & $21(53.8)$ & \\
\hline Sarcoidosis & 1 & 2 & \\
\hline Infective/inflammatory & 19 & 14 & \\
\hline Benign/no abnormality detected & 10 & 4 & \\
\hline Other & 4 & 1 & \\
\hline EBUS TBNA & 14 & 14 & \\
\hline Final diagnosis: malignant & $6(42.9)$ & 9 (64.3) & 0.45 \\
\hline Nonsmall cell lung cancer & 6 & 7 & \\
\hline Small cell lung cancer & 0 & 0 & \\
\hline Lymphoma & 0 & 0 & \\
\hline Metastatic malignancy & 0 & 2 & \\
\hline Final diagnosis: nonmalignant & $8(57.1)$ & 5 (35.7) & \\
\hline Sarcoidosis & 1 & 2 & \\
\hline Reactive (adequate sample) & 7 & 3 & \\
\hline
\end{tabular}

Regarding perceived willingness to undergo repeat procedure, proceduralists identified six subjects, including three of the five who had individually expressed unwillingness and three other subjects who expressed willingness to have a repeat procedure. Nurses identified 22 subjects, including four of the five who had expressed unwillingness and 18 other subjects who expressed willingness to have a repeat procedure. The similar number of willing subjects who were incorrectly identified and unwilling subjects who were not identified by proceduralists resulted in Bowker's test of symmetry demonstrating that proceduralist disagreement was not significantly asymmetric $(\mathrm{p}=0.65)$, whereas it showed asymmetric disagreement between nurses and subjects $(\mathrm{p}<0.01)$.

\section{Sedation assessments}

As expected, sedation scores (mOAA/S scale) were significantly different between the conscious sedation and general anaesthesia groups (table 2), with conscious sedation subjects perceived to be less sedated. Regression analysis showed no correlation between either proceduralist or nursing assessment of sedation 


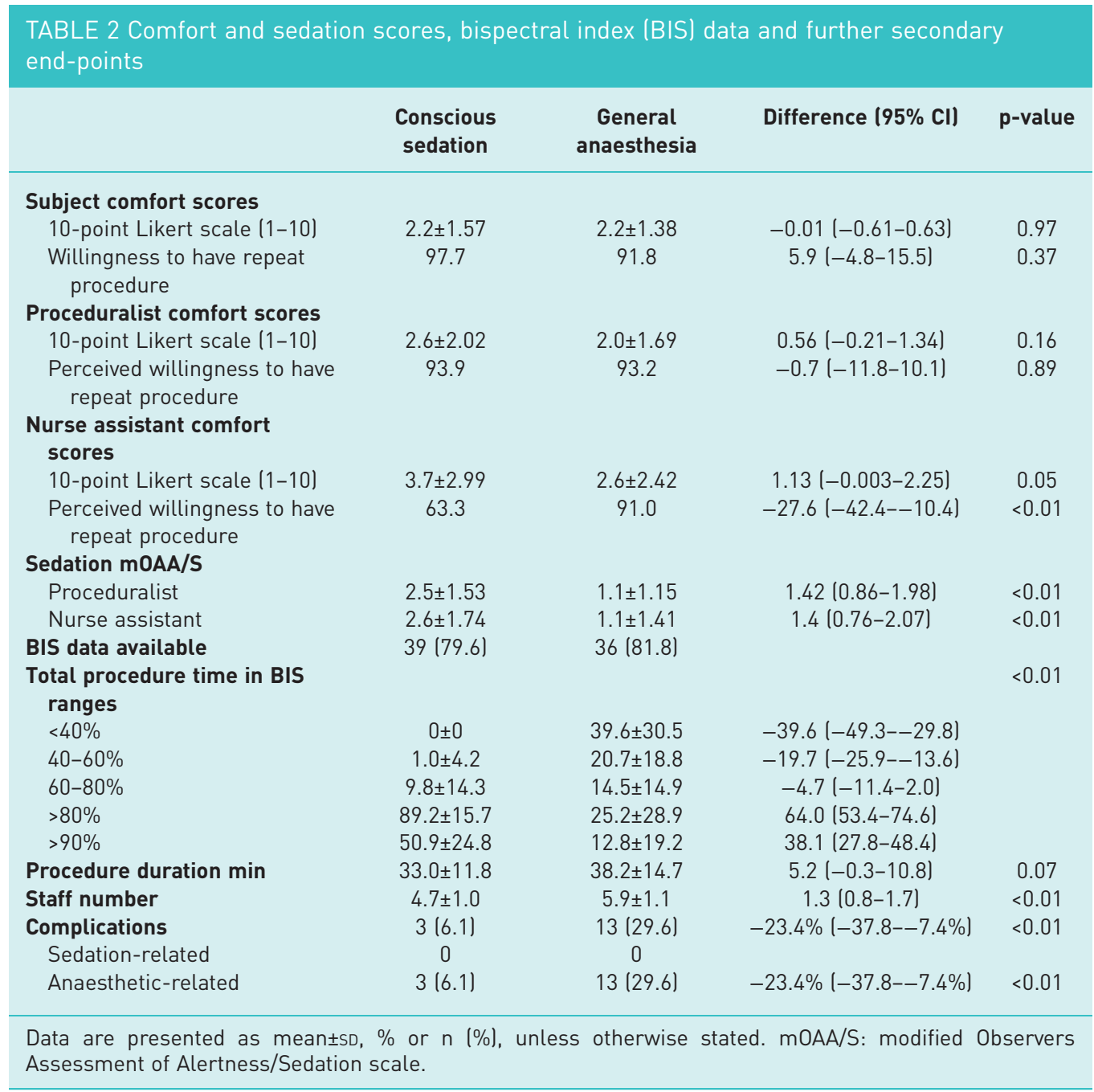

and subject comfort; the regression coefficients were 0.051 (95\% CI $-0.153-0.254)$ and 0.067 (95\% CI $-0.112-0.245)$, respectively.

Acceptable BIS data were available for $79.6 \%$ and $81.8 \%$ of subjects in the conscious sedation and general anaesthesia groups, respectively (table 2). As expected, there was a significantly different BIS distribution between the two groups (figure 3). The conscious sedation group spent a significantly larger proportion of time with BIS $>80(89.2 \%$ versus $29.2 \%, \mathrm{p}<0.01)$. The general anaesthesia group was more variable. Only $20.7 \%$ of time under general anaesthesia was spent in the "general anaesthesia target range" of 40-60, and $39.6 \%$ was spent in the "over-sedated" range of $<40$, compared to $1.0 \%$ and $0.0 \%$, respectively, with conscious sedation $(\mathrm{p}<0.01)$.

There was no significant relationship between percentage of procedure or time with BIS $<90$ and subject-reported comfort $(\mathrm{p}=0.868$ for time $>90 \mathrm{~min})$ Both proceduralist and nursing assessment of sedation correlated with percentage of BIS $>80(\mathrm{p}<0.01)$ (figure 4 and supplementary data).

\section{Other end-points}

Four procedures were not completed: three in the conscious sedation group and one in the general anaesthesia group. In the conscious sedation group two subjects did not tolerate the procedure, necessitating termination, and one case was cancelled due to equipment failure. The general anaesthesia procedure was aborted due to arrhythmia.

Procedure duration was not significantly different between conscious sedation and general anaesthesia $(33.0 \pm 11.8 \mathrm{~min}$ versus $38.2 \pm 14.7 \mathrm{~min}, \mathrm{p}=0.07)$. There were fewer staff present in procedures using conscious sedation $(4.7 \pm 1.0$ versus $5.9 \pm 1.1, \mathrm{p}<0.01)$. 

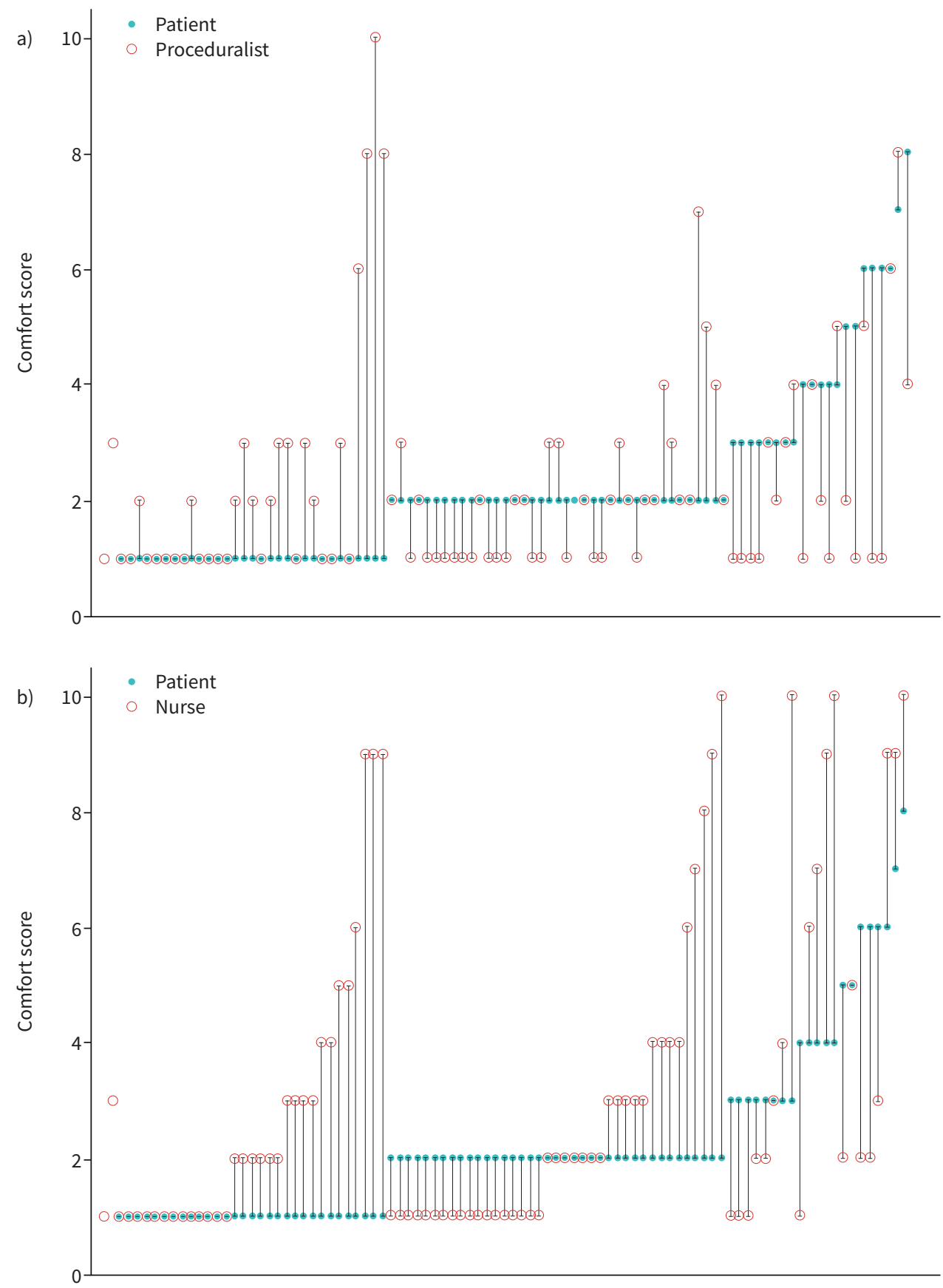

FIGURE 2 Matched-pairs analysis of 10-point Likert scale of comfort (higher score indicates more discomfort; each line represents one subject, sorted in ascending subject rating, then nurse/proceduralist rating). a) Subject assessment versus proceduralist's perception; b) subject assessment versus nurse's perception.

There were significantly more complications in the general anaesthesia group (29.6\%, 95\% CI $18.2-44.2 \%$ versus $6.1 \%$, 95\% CI 2.1-16.5\% with conscious sedation; $\mathrm{p}<0.01$ ) (table 2). All complications recorded were sedation-related. Aside from one case of arrhythmia in each group and a case of hypoxaemia in the general anaesthesia group, all other complications within both treatment arms were hypotension. Subjects whose BIS was $<40$ at any point in the procedure were more likely to have complications (OR $6.26,95 \%$ CI 1.76-22.34).

While noting that this study was not powered for these outcomes, in all diagnostic bronchoscopies there was no difference in diagnostic yield $(72.3 \%$ with conscious sedation versus $87.2 \%$ with general anaesthesia, $\mathrm{p}=0.11$ ). When assessing for possible malignancy, sensitivity, NPV and diagnostic accuracy were not different between the groups. Diagnostic accuracy with conscious sedation was 93.9\% (95\% CI 
FIGURE 3 Least-squares (LS) means plot of time at bispectral index (BIS) levels from randomeffects repeated-measures analysis.

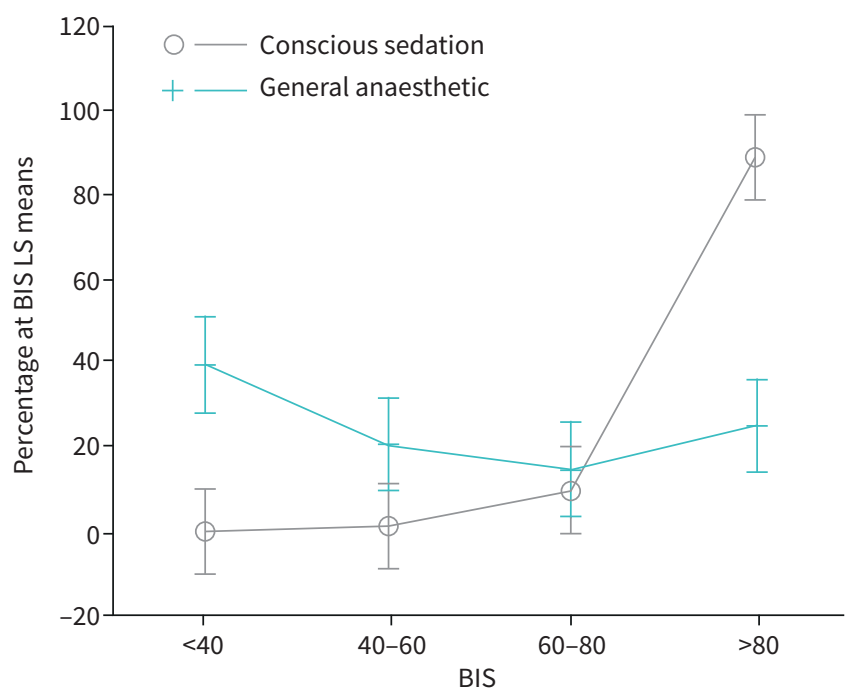

80.4-98.3\%), and with general anaesthesia was 86.5\% (95\% CI 72.0-94.1\%) ( $p=0.43)$. Fourteen EBUS TBNA procedures were included in each group, with all procedures completed and no significant difference in final diagnosis or diagnostic accuracy (table 3).

\section{Discussion}

This randomised trial confirms that conscious sedation is not inferior to general anaesthesia in providing patient comfort during bronchoscopy. Whereas some longer and more complex procedures may require general anaesthesia, this data along with other published research implies that the decision to use general anaesthesia need not be based on concerns about patient comfort, patient safety or diagnostic accuracy.

In addition, our study showed the novel data that the proceduralist's perception of subject comfort was not significantly different between conscious sedation and general anaesthesia, while nursing assessment of comfort was significantly less favourable under conscious sedation, with nurse assistants scoring subject comfort more poorly than the individual subjects themselves. Furthermore, nurses incorrectly identified a disproportionately higher number of subjects who they felt would not be willing to return, but who in fact were willing.

The proceduralists' and nurses' perception of subject comfort correlated more closely with each other than either did with subjects' own scores. This may suggest that while some patients may appear to be uncomfortable at times in a procedure, this does not always equate to any post-procedure feelings of discomfort, or, more importantly, reluctance to have repeated procedures.

Prior studies assessing subject comfort with differing methods of sedation have had conflicting conclusions. A randomised prospective trial of conscious sedation versus deep sedation assessing presence

FIGURE 4 Regression analysis of percentage time with bispectral index $(\mathrm{BIS})>80$ versus proceduralist sedation score (modified Observers Assessment of Alertness/Sedation (mOAA/S) scale).

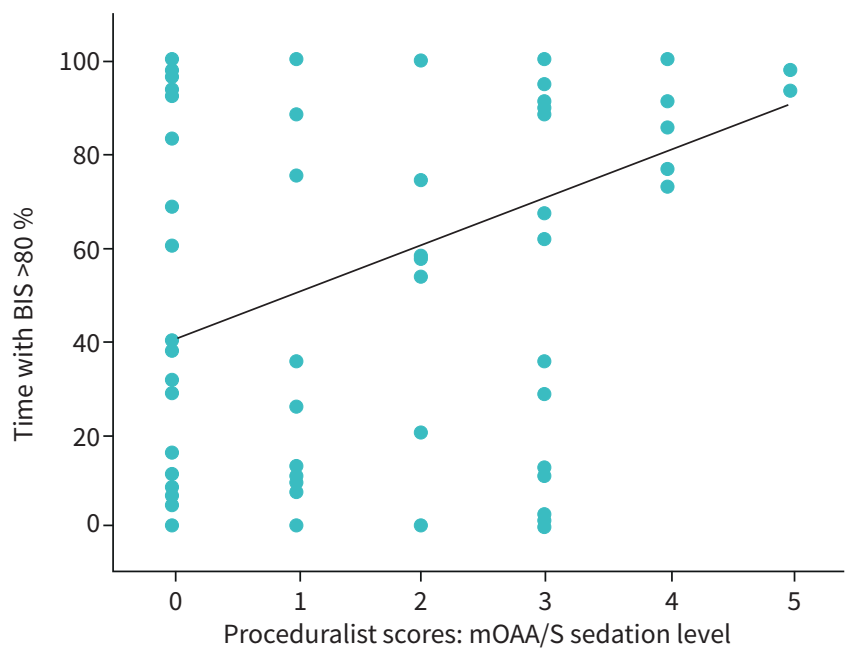




\begin{tabular}{|c|c|c|c|}
\hline & Conscious sedation & General anaesthesia & p-value \\
\hline All diagnostic procedures & 47 & 39 & \\
\hline Procedure not completed & 3 & 1 & 0.62 \\
\hline Diagnostic yield & 34 (72.3) & 34 (87.2) & 0.11 \\
\hline Diagnostic procedures assessing for malignancy & 33 & 37 & \\
\hline Procedure not completed & 1 & 1 & \\
\hline Lost to follow-up & 1 & 0 & \\
\hline Sensitivity $\%$ & 83.3 & 75.0 & \\
\hline NPV \% & 91.3 & 77.3 & \\
\hline Diagnostic accuracy $\%(95 \% \mathrm{CI})$ & $93.9(80.4-98.3)$ & $86.5(72.0-94.1)$ & 0.43 \\
\hline EBUS TBNA & 14 & 14 & \\
\hline Procedure not completed & 0 & 0 & \\
\hline Lost to follow-up & 0 & 0 & \\
\hline Nodal stations sampled & & & 0.28 \\
\hline Paratracheal $(2,4)$ & $11(46)$ & $5(24)$ & \\
\hline Subcarinal (7) & $4(17)$ & $6(29)$ & \\
\hline Hilar $(10,11,12)$ & $9(38)$ & $10(48)$ & \\
\hline Total & 24 & 21 & \\
\hline Passes per node & $3.28 \pm 0.8$ & $3.00 \pm 1.0$ & 0.29 \\
\hline Sensitivity \% & 100 & 87.5 & \\
\hline NPV \% & 100 & 85.7 & \\
\hline Diagnostic accuracy \% & 100 & 92.9 & 0.39 \\
\hline
\end{tabular}

or absence of distress with a post-procedure questionnaire suggests more subjects experience distress under conscious sedation [14]. However, as identified by the authors, the use of a binary question in this study may not be as precise as a linear scale, such as that used in our trial. Studies which have assessed subject comfort with differing sedation methods using a linear scale (usually a VAS) have all done so as a secondary outcome measure, and have not shown a significant difference $[15,16]$. This is consistent with our results.

Conscious sedation subjects had adequate comfort, despite being less sedated (as expected), as demonstrated by both proceduralist and nursing sedation scores as well as BIS levels. This supports recent guidelines that recommend either conscious sedation or deeper sedation for bronchoscopy $[8,35,36]$.

With respect to BIS, the noninferior comfort, subject satisfaction and diagnostic accuracy in the conscious sedation group suggests that the BIS levels achieved in our conscious sedation group are adequate for accomplishing an effective bronchoscopy. Furthermore, there was a significant relationship between BIS levels $<40$ at any time in the procedure and complications, particularly hypotension. This may suggest a role for BIS monitoring during general anaesthesia bronchoscopy to prevent over-sedation and complications. This is in keeping with a recent randomised cohort study of EBUS TBNA with and without BIS monitoring, which demonstrated fewer complications with BIS monitoring [33].

While procedure duration was not significantly different, conscious sedation was associated with lower staffing levels. This may result in reduced costs.

CASAL et al. [15] showed higher rates of complications in the conscious sedation group, but this was driven by excessive cough, inadequate sedation and hypertension. In the same study, hypotension occurred more commonly in the general anaesthesia group, matching our results $[15,16]$.

While our study was not powered to assess this outcome, our data suggest that conscious sedation is similar to general anaesthesia in terms of diagnostic accuracy, including EBUS TBNA from a wide range of nodal stations. This is in keeping with several recent trials assessing this question [15, 16, 20-23].

There are several limitations of this study to discuss. Subject blinding could possibly be achieved by having the anaesthetist present for all procedures; however, this was not practical within resource constraints in our unit. We accounted, at least in part, for the psychological impact this may have on subjects by demonstrating that pre- and post-procedure emotional states in the two groups were not significantly different. We excluded patients whom the treating physician felt had a tendency towards severe cough; this 
may have improved overall tolerance of conscious sedation. BIS data were lacking in $\sim 20 \%$ of cases, which is higher than may have been expected. This is likely due to the fact that BIS data were collected blindly, without concurrent anaesthetist monitoring, meaning that any issues with the trace would not have been detected, or resolved, at the time of the procedure. The amount of BIS data missing was equal between groups, and therefore we surmise that it was unlikely to have had a significant impact on the results.

Assessing the comfort of patients following anaesthesia is intrinsically challenging due to the amnesic effects of sedatives, with post-procedure subjective patient assessment remaining the most accepted assessment tool $[15,16,37-42]$. We followed recommendations to perform assessments within $48 \mathrm{~h}$ of the procedure to improve reliability of the patient response $[43,44]$.

\section{Conclusion}

In this prospective randomised trial, we have demonstrated that patient comfort with conscious sedation is noninferior to anaesthetist-controlled general anaesthesia for bronchoscopy. BIS monitoring confirmed increased complications when BIS descends $<40$. Nursing assessment tended to overemphasise patient discomfort compared to physicians. This study supports the flexibility and utility of having a conscious sedation approach, which is especially important in centres with limited anaesthetic support.

Acknowledgement: This study was performed at The Royal Brisbane and Women's Hospital, Queensland, Australia; the authors would like to acknowledge the significant contribution from the nurses of the Royal Brisbane and Women's Hospital bronchoscopy unit. Data from this study have been presented at the Thoracic Society of Australia and New Zealand annual scientific meetings in 2015, by Joseph Churton, and in 2018, by Thomas R. Skinner.

Author contributions: Thomas R. Skinner: literature search, acquisition, analysis and interpretation of data, manuscript preparation, review of the manuscript, and agreement to be accountable for all aspects of the work. Joseph Churton and David I. Fielding: literature search, conception and design of the work, acquisition, analysis, and interpretation of data, manuscript preparation, review of the manuscript, and agreement to be accountable for all aspects of the work. Timothy P. Edwards, Farzad Bashirzadeh, Christopher Zappala, Justin T. Hundloe, Hau Tan, Andrew J. Pattison and Maryann Todman: conception and design of the work, acquisition of data, review of the manuscript, and agreement to be accountable for all aspects of the work. Gunter F. Hartel: analysis, and interpretation of data, manuscript preparation, review of manuscript, and agreement to be accountable for all aspects of the work.

Conflict of interest: None declared.

\section{References}

1 Putinati S, Ballerin L, Corbetta L, et al. Patient satisfaction with conscious sedation for bronchoscopy. Chest 1999; 115: $1437-1440$.

2 Maguire GP, Rubinfeld AR, Trembath PW, et al. Patients prefer sedation for fibreoptic bronchoscopy. Respirology 1998; 3: 81-85.

3 Gonzalez R, De-La-Rosa-Ramirez I, Maldonado-Hernandez A, et al. Should patients undergoing a bronchoscopy be sedated? Acta Anaesthesiol Scand 2003; 47: 411-415.

4 Matot I, Kramer MR. Sedation in outpatient bronchoscopy. Respir Med 2000; 94: 1145-1153.

5 Stolz D, Chhajed PN, Leuppi JD, et al. Cough suppression during flexible bronchoscopy using combined sedation with midazolam and hydrocodone: a randomised, double blind, placebo controlled trial. Thorax 2004; 59: 773-776.

6 British Thoracic Society Bronchoscopy Guidelines Committee, a Subcommittee of the Standards of Care Committee of the British Thoracic Society. British Thoracic Society guidelines on diagnostic flexible bronchoscopy. Thorax 2001; 56: Suppl. 1, i1-i21.

7 Du Rand IA, Blaikley J, Booton R, et al. Summary of the British Thoracic Society guideline for diagnostic flexible bronchoscopy in adults. Thorax 2013; 68: 786-787.

8 Wahidi MM, Herth F, Yasufuku K, et al. Technical aspects of endobronchial ultrasound-guided transbronchial needle aspiration: CHEST guideline and expert panel report. Chest 2016; 149: 816-835.

9 American Society of Anesthesiologists Task Force on Sedation and Analgesia by Non-Anesthesiologists. Practice guidelines for sedation and analgesia by non-anesthesiologists. Anesthesiology 2002; 96: 1004-1017.

10 Kern M, Kerner T, Tank S. Sedation for advanced procedures in the bronchoscopy suite: proceduralist or anesthesiologist? Curr Opin Anaesthesiol 2017; 30: 490-495.

11 McCambridge AJ, Boesch RP, Mullon JJ. Sedation in bronchoscopy: a review. Clin Chest Med 2018; 39: 65-77.

12 Smyth CM, Stead RJ. Survey of flexible fibreoptic bronchoscopy in the United Kingdom. Eur Respir J 2002; 19: 458-463.

13 Barnett AM, Jones R, Simpson G. A survey of bronchoscopy practice in Australia and New Zealand. J Bronchology Interv Pulmonol 2016; 23: 22-28.

14 Haga T, Fukuoka M, Morita M, et al. A prospective analysis of the efficacy and complications associated with deep sedation with midazolam during fiberoptic bronchoscopy. J Bronchology Interv Pulmonol 2016; 23: 106-111.

15 Casal RF, Lazarus DR, Kuhl K, et al. Randomized trial of endobronchial ultrasound-guided transbronchial needle aspiration under general anesthesia versus moderate sedation. Am J Respir Crit Care Med 2015; 191: 796-803.

16 Fernandes MGO, Santos VF, Martins N, et al. Endobronchial ultrasound under moderate sedation versus general anesthesia. J Clin Med 2018; 7: 421.

17 Yasufuku K, Chiyo M, Sekine Y, et al. Real-time endobronchial ultrasound-guided transbronchial needle aspiration of mediastinal and hilar lymph nodes. Chest 2004; 126: 122-128. 
18 Herth FJ, Eberhardt R, Vilmann P, et al. Real-time endobronchial ultrasound guided transbronchial needle aspiration for sampling mediastinal lymph nodes. Thorax 2006; 61: 795-798.

19 Sarkiss M, Kennedy M, Riedel B, et al. Anesthesia technique for endobronchial ultrasound-guided fine needle aspiration of mediastinal lymph node. J Cardiothorac Vasc Anesth 2007; 21: 892-896.

20 Aswanetmanee $\mathrm{P}$, Limsuwat $\mathrm{C}$, Kabach $\mathrm{M}$, et al. The role of sedation in endobronchial ultrasound-guided transbronchial needle aspiration: systematic review. Endosc Ultrasound 2016; 5: 300-306.

21 Franzen D, Schneiter D, Weder W, et al. Impact of sedation technique on the diagnostic accuracy of endobronchial ultrasound-guided transbronchial needle aspiration. Endosc Ultrasound 2017; 6: 257-263.

22 Öztas S, Aka Aktürk U, Alpay LA, et al. A comparison of propofol-midazolam and midazolam alone for sedation in endobronchial ultrasound-guided transbronchial needle aspiration: a retrospective cohort study. Clin Respir 2017; 11: 935-941.

23 Cornelissen CG, Dapper J, Dreher M, et al. Endobronchial ultrasound-guided transbronchial needle aspiration under general anesthesia versus bronchoscopist-directed deep sedation: a retrospective analysis. Endosc Ultrasound 2019; 8: 204-208.

24 Chernik DA, Gillings D, Laine $\mathrm{H}$, et al. Validity and reliability of the Observer's Assessment of Alertness/Sedation Scale: study with intravenous midazolam. J Clin Psychopharmacol 1990; 10: 244-251.

25 Fadaizadeh L, Hoseyni MS, Shajareh E, et al. Use of bispectral index score for interventional bronchoscopy procedures. Tanaffos 2015; 14: 246-251.

26 Glass PS, Bloom M, Kearse L, et al. Bispectral analysis measures sedation and memory effects of propofol, midazolam, isoflurane, and alfentanil in healthy volunteers. Anesthesiology 1997; 86: 836-847.

27 Bower AL, Ripepi A, Dilger J, et al. Bispectral index monitoring of sedation during endoscopy. Gastrointest Endosc 2000; 52: 192-196.

28 von Delius S, Thies P, Rieder T, et al. Auditory evoked potentials compared with bispectral index for monitoring of midazolam and propofol sedation during colonoscopy. Am J Gastroenterol 2009; 104: 318-325.

29 Das S, Ghosh S. Monitored anesthesia care: an overview. J Anaesthesiol Clin Pharmacol 2015; 31: 27-29.

30 Weaver CS, Hauter WH, Duncan CE, et al. An assessment of the association of bispectral index with 2 clinical sedation scales for monitoring depth of procedural sedation. Am J Emerg Med 2007; 25: 918-924.

31 Chisholm CJ, Zurica J, Mironov D, et al. Comparison of electrophysiologic monitors with clinical assessment of level of sedation. Mayo Clin Proc 2006; 81: 46-52.

32 Schlatter L, Pflimlin E, Fehrke B, et al. Propofol versus propofol plus hydrocodone for flexible bronchoscopy: a randomised study. Eur Respir J 2011; 38: 529-537.

33 Quesada N, Júdez D, Martínez Ubieto J, et al. Bispectral index monitoring reduces the dosage of propofol and adverse events in sedation for endobronchial ultrasound. Respiration 2016; 92: 166-175.

34 Clark G, Licker M, Younossian AB, et al. Titrated sedation with propofol or midazolam for flexible bronchoscopy: a randomised trial. Eur Respir J 2009; 34: 1277-1283

35 Yıldırım F, Özkaya Ş, Yurdakul AS. Factors affecting patients' comfort during fiberoptic bronchoscopy and endobronchial ultrasound. J Pain Res 2017; 10: 775-781.

36 González Aguirre JE, Chavarría Martínez U, Rodríguez Mier D, et al. Bronchoscope insertion route and patient comfort during flexible bronchoscopy. Int J Tuberc Lung Dis 2015; 19: 356-361.

37 Dreher M, Ekkernkamp E, Storre JH, et al. Sedation during flexible bronchoscopy in patients with pre-existing respiratory failure: midazolam versus midazolam plus alfentanil. Respiration 2010; 79: 307-314.

38 Ekkelenkamp VE, Dowler K, Valori RM, et al. Patient comfort and quality in colonoscopy. World J Gastroenterol 2013; 19: 2355-2361.

39 Rostom A, Ross ED, Dubé C, et al. Development and validation of a nurse-assessed patient comfort score for colonoscopy. Gastrointest Endosc 2013; 77: 255-261.

40 Celebi D, Yllmaz E, Sahin ST, et al. The effect of music therapy during colonoscopy on pain, anxiety and patient comfort: a randomized controlled trial. Complement Ther Clin Pract 2020; 38: 101084.

41 Steinfort DP, Irving LB. Patient satisfaction during endobronchial ultrasound-guided transbronchial needle aspiration performed under conscious sedation. Respir Care 2010; 55: 702-706.

42 Jeyabalan A, Medford AR. Endobronchial ultrasound-guided transbronchial needle aspiration: patient satisfaction under light conscious sedation. Respiration 2014; 88: 244-250.

43 Auquier P, Pernoud N, Bruder N, et al. Development and validation of a perioperative satisfaction questionnaire. Anesthesiology 2005; 102: 1116-1123.

44 Leffler DA, Bukoye B, Sawhney M, et al. Development and validation of the PROcedural Sedation Assessment Survey (PROSAS) for assessment of procedural sedation quality. Gastrointest Endosc 2015; 81: 194-203. 